

\title{
Factors affecting successful atropine therapy for infantile hypertrophic pyloric stenosis: a retrospective analysis using a nationwide database in Japan
}

\author{
Michimasa Fujiogi ${ }^{1,2}$, Nobuaki Michihata ${ }^{3}$, Hiroki Matsui ${ }^{2}$, Kiyohide Fushimi ${ }^{4}$, Hideo Yasunaga ${ }^{2}$, \\ Tetsuya Ishimaru ${ }^{5}$, Jun Fujishiro ${ }^{1}$
}

\section{ABSTRACT}

BACKGROUND

Intravenous atropine therapy (IAT) is feasible for infantile hypertrophic pyloric stenosis (IHPS). Currently, IAT is recommended for only patients with risks against surgery. However, reported proportions of successful IAT ranged widely, being from 70 to $90 \%$. Factors related to successful IAT remain unknown. This study aimed to identify predictive factors of successful IAT for IHPS using a national inpatient database in Japan.

METHODS

Using the Diagnosis Procedure Combination database, we identified children with IHPS from July 2010 to March 2016. We compared patients' characteristics between the IAT and surgery groups, and between the successful and unsuccessful IAT groups. Multivariable logistic regression analysis was performed for successful IAT.

\section{RESULTS}

We identified 1,822 eligible patients (526 IAT patients). IAT had a success rate of $73.4 \%$ $(\mathrm{n}=386)$ and 140 unsuccessful patients subsequently underwent surgery. Body weight at admission in the successful group was significantly heavier than that in the unsuccessful group. The Multivariate logistic analysis showed that the proportion of successful IATs was significantly higher in the heavy weight $(4,000-4,999 \mathrm{~g}$ ) (odds ratio, 2.03; 95\% confidence interval, 1.32-3.13) and very heavy weight ( $\geq 5,000$ g) groups (odds ratio, 5.35 ; $95 \%$ confidence interval, 1.80-15.9) than in the middle weight (3,000-3,999 g) group.

CONCLUSIONS

Heavier body weight was associated with successful IAT. These results are useful for patients select therapeutic options for IHPS, including surgery and IAT.

KEY WORDS

atropine therapy, hypertrophic pyloric stenosis, pediatrics, predictive factor, pyloromyotomy
${ }^{1}$ Department of Pediatric Surgery, Graduate School of Medicine, The University of Tokyo ${ }^{2}$ Department of Clinical Epidemiology and Health Economics, School of Public Health, The University of Tokyo

${ }^{3}$ Department of Health Services Research, Graduate School of Medicine, The University of Tokyo

${ }^{4}$ Department of Health Policy and Informatics, Tokyo Medical and Dental University Graduate School

${ }^{5}$ Department of Pediatric Surgery, Saitama Children's Medical Center

Corresponding author: Jun Fujishiro

Department of Pediatric Surgery, Graduate School of Medicine, The University of Tokyo, 7-3-1 Hongo, Bunkyo-ku, Tokyo 113-0033, Japan.

E-mail: jfujishi-tky@umin.ac.jp

Received: January 16, 2019

Accepted: February 9, 2019

No.19-03

(C) 2019 Society for Clinical Epidemiology 


\section{INTRODUCTION}

I nfantile hypertrophic pyloric stenosis (IHPS) is a condition characterized by hypertrophy of the circular muscle of the pylorus, causing narrowing and elongation of the pyloric channel. The standard treatment for IHPS is extramucosal pyloromyotomy. This procedure has remained unimproved for a long time, except for minimally invasive access modifications that include circumumbilical incision [1] and the laparoscopic approach [2].

The effectiveness of non-surgical treatment, intravenous atropine therapy (IAT), for IHPS has mainly been reported from Japan [3-9]. Previous studies have reported that IAT is performed in approximately $30 \%$ of patients with IHPS in Japan [7]. Avoidance of surgery is the potential merit of IAT for patients and patients' families. However, IAT has not gained wide acceptance in Western countries because IAT is not effective for all patients and is associated with a prolonged length of hospital stay [10]. Therefore, it is recommended that the IAT is reserved only in selected patients, such as those at high risk for general anesthesia or surgical procedures $[10,11]$. Reported proportions of success of IAT ranged widely, being from 70 to $90 \%$ [4-8].

Several studies showed no significant differences in imaging, laboratory data (acid-base balance of venous blood gas, serum chloride, and serum potassium), clinical symptoms at presentation such as pyloric thickness and length; and duration of symptoms before admission between successful and unsuccessful IAT groups $[4,6$, 12]. However, association between patients' characteristic (such as body status and congenital malformation) and successful IAT remains controversial. A previous cohort study showed that successful IAT group had a significant heavier body weight than the unsuccessful IAT group, even though age was not significantly associated with successful IAT. [4]. Another study showed there was no significant difference in body weight at admission between 2 groups [6].

To address these knowledge gaps, this study aimed to compare patients' characteristics between the successful and unsuccessful IAT groups and to identify predictive factors of successful IAT for IHPS using a national inpatient database in Japan.

\section{METHODS}

DATA SOURCE

This study was a retrospective, nationwide, observational study that used the Diagnosis Procedure Combination (DPC) database. All 82 academic hospitals are obliged to participate in this database, but participation by community hospitals is voluntary. The database included the following data: unique identifiers of hospitals; patients' age, body weight, body height, and sex; emergent or elective admission; diagnoses, comorbidities at admission, and complications after admission recorded with text data in the Japanese language and the International Classification of Diseases, Tenth Revision (ICD-10), codes [13]; procedures; drugs; devices; length of stay (LOS); and discharge status. Vital signs, current symptoms, and laboratory data for each patient are not included in the DPC database. Discharge abstract data for each patient are recorded at discharge by the attending physicians. A previous study showed that the validity of diagnoses and procedure records in the DPC database was high in general [14].

The present study was based on a secondary analysis of the administrative claims data. The requirement for informed consent was waived because of the anonymous nature of the data. Study approval was obtained from the Institutional Review Board at The University of Tokyo.

\section{STUDY POPULATION}

We identified the pediatric population (0-100 days old) who were admitted with a diagnosis of IHPS between July 2010 and March 2016. IHPS was identified from the Japanese text-based diagnosis of IHPS and the specific ICD-10 code, Q400. Patients were identified with the diagnosis of IHPS and management with IHPS-specific treatments (atropine and/or pyloromyotomy). We excluded patients whose outcome data were missing (e.g., transfer to another hospital). We also excluded those who were treated with the oral form of atropine for initial treatment.

\section{MEASUREMENTS OF VARIABLES}

The following patients' characteristics were collected: sex, age, body weight at admission, congenital malformations, chronic medical conditions (heart, kidney, pulmonary, endocrine, gastrointestinal, neurological diseases, cancer or leukemia, and autoimmune diseases), and hospital volume. Body weight at admission was classified into the following four groups: group A, $\leq 2,999$ g; B, 3,0003,999 g; C, 4,000-4,999 g; and D, $\geq 5,000$ g. Age was classified into the following three groups: $<28,28-59$, or $\geq 60$ days old. We also examined the following congenital malformations: circulatory system, (ICD-10 code, Q20Q28), respiratory system (Q30-Q34), digestive system 
(Q38-Q45), and chromosomal abnormalities (Q90Q99). The number of chronic medical conditions was defined according to the pediatric complex chronic conditions classification system version 2 [15]. The number of complex chronic comorbidities was classified as zero, one, or $\geq$ two. Hospital volume was defined as the average annual number of IATs for IHPS performed at each hospital and was categorized into $<$ three or $\geq$ three times per year.

Successful IAT was defined as discharge without surgical intervention and readmission (within 30 days from discharge) for surgical intervention. Surgical success was defined as discharge without complications requiring reoperation and without atropine therapy (intravenous or oral) after surgery (including readmission). Complications included bleeding (ICD-10 codes: T810, T811, K661), surgical site infection (T793, T814), peritonitis or peritoneal abscess (K65), ileus (K56, K913), respiratory complications (J14-18, J95.8, J95.9, J96.0, J96.9), urinary tract infection (N10, N30, N39.0), shock (R57), and gastric or duodenal perforation (S363-4, K631).

\section{STATISTICAL ANALYSES}

We compared the patients' background, LOS, and complications after admission between the IAT and surgery groups. We used Fisher's exact test and the chi-square test to compare proportions for categorical variables (e.g., sex), and the $t$-test and Mann-Whitney $U$ test to compare means or medians for continuous variables (e.g., age). We compared the patients' background between the successful and unsuccessful IAT groups. We also conducted a trend analysis by the Cochran-Armitage test to compare the association between body weight and the success rate $[16,17]$. We performed multivariable logistic regression analysis for successful IAT with adjustment for characteristics of patients and hospitals. To account for clustering of patients within hospitals, the logistic regression model was fitted with a generalized estimating equation. The covariates included age at admission, sex, body weight at admission, congenital malformations, the number of complex chronic conditions, and annual hospital volume for IAT. Missing data are described in Table 1, and patients with incomplete data were excluded from the regression model. We used a significance level of $\mathrm{p}<0.05$ for all statistical tests, and all reported $\mathrm{p}$ values were two-sided. All statistical analyses were conducted using Stata/MP 14.0 (Stata Corp., College Station, TX, USA).

\section{SENSITIVITY ANALYSIS}

We excluded patients transferred to other hospital in the main analysis. However, if majority of patients who underwent IAT were transferred to other hospital to receive surgery, the results might be biased. We therefore classified patients transferred to other hospital to the unsuccessful group, and performed another multivariable logistic regression analysis for successful IAT as a sensitivity analysis.

\section{RESULTS}

We identified 1,906 patients with IHPS who received atropine or pyloromyotomy during the study period. We excluded patients whose outcome data were missing $(n=67)$ and those who were treated with the oral form of atropine for initial treatment $(n=17)$. Therefore, we identified 1,822 eligible patients, including 526 (29\%) patients with IAT and 1,296 (71\%) patients who underwent surgery.

There were no significant differences in age, sex, and body weight at admission between the IAT and surgery groups. Patients with congenital malformations (circulatory system and digestive system) and those with complex chronic conditions were significantly less likely to receive surgery (Table $\mathbf{1}$ )

IAT was successful in 386 of 526 (73.4\%) patients. All patients with unsuccessful IAT $(\mathrm{n}=140)$ subsequently underwent an operation for IHPS. Surgery was successful in $98.8 \%$ of the patients; reoperation was performed in four $(0.3 \%)$, and postoperative atropine therapy (intravenous or oral form) was performed for $11(0.9 \%)$ patients. The median (interquartile range) LOS was 13 days (9-17 days) in the IAT group and 8 days (6-10 days) in the surgery group. The LOS was significantly longer in the IAT group than in the surgery group $(\mathrm{p}<0.001)$. The proportion of complications was significantly lower in the IAT group than in the surgery group $(1.0 \%$ vs. $2.5 \%$, $\mathrm{p}=0.04)$.

Table 2 shows the patients' characteristics of IAT between the successful and unsuccessful IAT groups. The mean (standard deviation) age at admission was significantly older in the successful IAT group than in the unsuccessful IAT group (40(17) vs. 34(16) days, $\mathrm{p}<0.001)$. The mean body weight was significantly heavier in the successful IAT group than in the unsuccessful IAT group $(3,946(774) \mathrm{g}$ vs. 3,592(671) g, p < 0.001). Sex, congenital malformations, and the number of complex chronic conditions were similar in both groups.

There was a significant association between higher 
proportion of successful IAT and heavier body weight $(\mathrm{p}<0.001$, Fig. 1).

Table 3 shows the results of multivariable logistic regression analysis for successful IAT. With reference to group B, a higher proportion of successful IATs was significantly associated with heavier body weight at admission in groups $C$ (odds ratio, 2.03; 95\% confidence interval, 1.32-3.13) and D (odds ratio, 5.35; 95\% confidence interval, 1.80-15.9). Older patients tended to have a higher proportion of successful IATs, but this was not significant. Hospital volume was not significantly associated with successful IAT.

In the sensitivity analysis, with reference to group $\mathrm{B}$, a higher proportion of successful IATs was also significantly associated with heavier body weight at admission in groups C (odds ratio, 1.65; 95\% confidence interval, 1.14-2.39) and D (odds ratio, 3.01; 95\% confidence interval, 1.27-7.13).

\section{DISCUSSION}

This study, which used a large, nationwide inpatient database, showed that the proportion of successful IAT was $73.4 \%$ and weight at presentation was associated with successful IAT.

The definitive cause for IHPS remains unknown. A previous study reported that tonic and phasic pyloric contractions frequently occurred in patients with IHPS. Intravenous atropine suppressed such incoordination transiently [18]. However predictive factor affecting successful IAT is unknown. Several studies showed that no significant differences in duration of vomiting before admission; laboratory data including acid-base balance; and thickness and length of pyloric muscle between successful and unsuccessful IAT groups[4, 6, 12]. One study reported that a predictive factor of unsuccessful IAT was projectile vomiting that occurred more than five times by

\begin{tabular}{|c|c|c|c|c|c|c|c|}
\hline & \multicolumn{2}{|c|}{$\begin{array}{l}\text { Atropine therapy } \\
\qquad(\mathrm{n}=526)\end{array}$} & \multicolumn{2}{|c|}{$\begin{array}{c}\text { Surgery } \\
(\mathrm{n}=1,296)\end{array}$} & \multicolumn{2}{|c|}{$\begin{array}{c}\text { Total } \\
(\mathrm{n}=1,822)\end{array}$} & \multirow{2}{*}{$\mathrm{p}$ value } \\
\hline & $\mathrm{n}$ & (\%) & $\mathrm{n}$ & (\%) & $\mathrm{n}$ & (\%) & \\
\hline \multicolumn{8}{|l|}{ Sex } \\
\hline Male & 440 & $(83.7)$ & 1,073 & $(82.8)$ & 1,513 & $(83.0)$ & 0.66 \\
\hline \multicolumn{8}{|l|}{ Age at admission (days) } \\
\hline$<28$ & 141 & $(26.8)$ & 323 & $(24.9)$ & 464 & $(25.5)$ & 0.32 \\
\hline $28-59$ & 319 & $(60.7)$ & 833 & $(64.3)$ & 1,152 & $(63.2)$ & \\
\hline$\geq 60$ & 66 & $(12.6)$ & 140 & $(10.8)$ & 206 & $(11.3)$ & \\
\hline \multicolumn{8}{|l|}{ Body weight at admission (g) } \\
\hline Group A $(\leq 2,999)$ & 49 & (9.3) & 124 & (9.6) & 173 & (9.5) & 0.23 \\
\hline Group B $(3,000-3,999)$ & 256 & $(48.7)$ & 691 & $(53.3)$ & 947 & $(52.0)$ & \\
\hline Group C $(4,000-4,999)$ & 169 & $(32.1)$ & 380 & (29.3) & 549 & $(30.1)$ & \\
\hline Group D $(\geq 5,000)$ & 41 & (7.8) & 72 & (5.6) & 113 & $(6.2)$ & \\
\hline Missing & 11 & (2.1) & 29 & (2.2) & 40 & $(2.2)$ & \\
\hline \multicolumn{8}{|l|}{ Congenital malformations } \\
\hline Circulatory system & 22 & $(4.2)$ & 26 & (2.0) & 48 & (2.6) & 0.009 \\
\hline Respiratory system & 2 & $(0.4)$ & 2 & $(0.2)$ & 4 & $(0.2)$ & 0.33 \\
\hline Digestive system & 7 & $(1.3)$ & 5 & $(0.4)$ & 12 & $(0.7)$ & 0.048 \\
\hline Chromosomal abnormalities & 1 & $(0.2)$ & 0 & 0.0 & 1 & $(0.1)$ & 0.29 \\
\hline Number of complex chronic conditions & & & & & & & $<0.001$ \\
\hline 0 & 471 & $(89.5)$ & 1,226 & (94.6) & 1,697 & $(93.1)$ & \\
\hline 1 & 43 & (8.2) & 65 & (5.0) & 108 & (5.9) & \\
\hline$\geq 2$ & 12 & (2.3) & 5 & $(0.4)$ & 17 & $(0.9)$ & \\
\hline
\end{tabular}


Table 2 Comparison of clinical factors between the successful and unsuccessful IAT groups

\begin{tabular}{|c|c|c|c|c|c|}
\hline & \multicolumn{2}{|c|}{ Successful IAT $(\mathrm{n}=386)$} & \multicolumn{2}{|c|}{ Unsuccessful IAT $(\mathrm{n}=140)$} & \multirow{2}{*}{$\mathrm{p}$ value } \\
\hline & $\mathrm{n}$ & $(\%)$ & $\mathrm{n}$ & $(\%)$ & \\
\hline \multicolumn{6}{|l|}{ Sex } \\
\hline Male & 321 & $(83.2)$ & 119 & $(85.0)$ & 0.61 \\
\hline \multicolumn{6}{|l|}{ Age at admission (days) } \\
\hline$<28$ & 90 & $(23.3)$ & 51 & $(36.4)$ & 0.007 \\
\hline $28-59$ & 242 & $(62.7)$ & 77 & $(55.0)$ & \\
\hline$\geq 60$ & 54 & $(14.0)$ & 12 & $(8.6)$ & \\
\hline \multicolumn{6}{|l|}{ Body weight at admission (g) } \\
\hline Group A $(\leq 2,999)$ & 30 & $(7.8)$ & 19 & $(13.6)$ & 0.001 \\
\hline Group B $(3,000-3,999)$ & 176 & $(45.6)$ & 80 & $(57.1)$ & \\
\hline Group C $(4,000-4,999)$ & 136 & $(35.2)$ & 33 & $(23.6)$ & \\
\hline Group D $(\geq 5,000)$ & 37 & $(9.6)$ & 4 & (2.9) & \\
\hline Missing & 7 & $(1.8)$ & 4 & $(2.9)$ & \\
\hline \multicolumn{6}{|l|}{ Congenital malformations } \\
\hline Circulatory system & 18 & $(4.7)$ & 4 & $(2.9)$ & 0.46 \\
\hline Respiratory system & 1 & $(0.3)$ & 1 & $(0.7)$ & 0.46 \\
\hline Digestive system & 5 & $(1.3)$ & 2 & $(1.4)$ & 1.00 \\
\hline Chromosomal abnormalities & 1 & $(0.3)$ & 0 & 0.0 & 1.00 \\
\hline Number of complex chronic conditions & & & & & 0.64 \\
\hline 0 & 348 & $(90.2)$ & 123 & $(87.9)$ & \\
\hline 1 & 30 & $(7.8)$ & 13 & $(9.3)$ & \\
\hline$\geq 2$ & 8 & $(2.1)$ & 4 & $(2.9)$ & \\
\hline Annual hospital volume of IAT for IHPS & & & & & 0.44 \\
\hline Low ( $<3$ cases $)$ & 323 & $(83.7)$ & 121 & $(86.4)$ & \\
\hline High ( $\geq 3$ cases) & 63 & $(16.3)$ & 19 & $(13.6)$ & \\
\hline
\end{tabular}






\begin{tabular}{|c|c|c|c|}
\hline \multirow{2}{*}{ Variables } & \multicolumn{2}{|c|}{ Successful IAT } & \multirow{2}{*}{$\mathrm{p}$ value } \\
\hline & Odds ratio & $95 \% \mathrm{CI}$ & \\
\hline \multicolumn{4}{|l|}{ Sex } \\
\hline Female & Ref & & \\
\hline Male & 0.99 & $0.57-1.73$ & 0.98 \\
\hline \multicolumn{4}{|l|}{ Age at admission (days) } \\
\hline$<28$ & Ref & & \\
\hline $28-59$ & 1.13 & $0.71-1.82$ & 0.60 \\
\hline$\geq 60$ & 0.87 & $0.42-1.82$ & 0.71 \\
\hline \multicolumn{4}{|l|}{ Weight at admission (g) } \\
\hline Group A $(\leq 2,999)$ & 0.70 & $0.31-1.57$ & 0.39 \\
\hline Group B $(3,000-3,999)$ & Ref & & \\
\hline Group C $(4,000-4,999)$ & 2.03 & $1.32-3.13$ & 0.001 \\
\hline Group D $(\geq 5,000)$ & 5.34 & $1.80-15.9$ & 0.003 \\
\hline \multicolumn{4}{|l|}{ Congenital malformations } \\
\hline Circulatory system & 1.80 & $0.62-5.2$ & 0.28 \\
\hline Respiratory system & 0.13 & $0.002-8.10$ & 0.33 \\
\hline Digestive system & 1.38 & $0.13-14.6$ & 0.79 \\
\hline \multicolumn{4}{|c|}{ Number of complex chronic conditions } \\
\hline 0 & Ref & & \\
\hline 1 & 1.17 & $0.55-2.48$ & 0.68 \\
\hline$\geq 2$ & 1.55 & $0.21-11.5$ & 0.67 \\
\hline \multicolumn{4}{|c|}{ Annual hospital volume of IAT for IHPS } \\
\hline Low $(<3)$ & Ref & & \\
\hline $\operatorname{High}(\geq 3)$ & 1.67 & $0.61-4.56$ & 0.32 \\
\hline
\end{tabular}

day 3 after starting intravenous atropine [6]. This may lead to the decision of converting IAT to surgery. However, this factor is not available for prediction of outcome before starting IAT.

Several studies on atropine treatment for IHPS compared body weight at admission between successful and unsuccessful IAT groups $[4,6,12]$. All of these studies reported a tendency that the successful IAT group had a heavier body weight than the unsuccessful IAT group. Although there was no detailed information on body weight in these studies, this finding supports our finding in the present study.

In Western countries, IAT is not generally recommended for patients with IHPS because of its lower success rate and longer LOS. Atropine therapy is recommended only for selected patients with a high risk for general anesthesia or surgical procedures [10, 11]. As suggested in these reports, patients with congenital malformations (circulatory system and digestive system) and complex chronic conditions were significantly more likely to receive IAT in the present study. However, IAT was also selected for patients without risks. IAT may have been selected according to not only the patient's condition but also other reason such as the wishes of the patient's family. Various complications of surgery were reported, including a complication rate of $1 \%$ in incomplete pyloromyotomy, $0.7 \%$ in mucosal perforation, and $1.5 \%$ in surgical site complications $[19,20]$. Because the reported complication rate is low, and the success rate is high, pyloromyotomy is the standard treatment for patients without risks.

It may be chosen IAT for patients without risks to 
avoid surgery in consideration of various reason (e.g., the wishes of the patient's family). Our results suggest that body weight at presentation can be a factor which should be taken into consideration for selecting IAT as the firstline treatment.

This study showed that there was no significant relationship between hospital volume and success of IAT after adjustment for characteristics of patients and hospitals. In Japan where IAT is frequently performed, IAT can also be adequately performed in low-volume hospitals. Therefore, the difference in treatment outcomes between hospitals may have been reduced.

Several limitations of this study should be acknowledged. First, we were unable to assess atropine-specific adverse events, such as tachycardia and flushing, because of the nature of the database. Reported proportions of atropine-specific adverse events were $0-33.3 \%$. However, IAT was associated with only minor complications[10]. Second, we did not know detailed treatment methods, such as dosing frequency, dosing interval, and amount of milk per one time because of the nature of the database. Third, although the database included approximately
$50 \%$ of all acute care inpatient data in Japan[14], participation in this database was voluntary for each institution, and patient selection was not based on a random sampling method. Hence, the generalizability of this results may be limited.

\section{CONCLUSION}

This retrospective, nationwide study in Japan where IAT is widely applied shows that heavier body weight was associated with successful IAT. We should choose either surgery or IAT for IHPS with consideration for weight at presentation as well as the risk of operation.

\section{ACKNOWLEDGMENTS}

This work was supported by grants from the Ministry of Health, Labour and Welfare, Japan (H29-Policy-Designated-009 and H29-ICT-General-004); and the Ministry of Education, Culture, Sports, Science and Technology, Japan (17H04141).

\section{CONFLICT OF INTEREST}

None declared.

REFERENCES

1. Tan KC, Bianchi A. Circumumbilical incision for pyloromyotomy. Br J Surg 1986;73:399. 2. Alain JL, Grousseau D, Terrier G. Extramucosal pyloromyotomy by laparoscopy. Surg Endosc 1991;5:174-5.

3. Nagita A, Yamaguchi J, Amemoto K, Yoden A, Yamazaki T, Mino M. Management and ultrasonographic appearance of infantile hypertrophic pyloric stenosis with intravenous atropine sulfate. J Pediatr Gastroenterol Nutr 1996;23:172-7.

4. Kawahara H, Takama Y, Yoshida H, Nakai H, Okuyama H, Kubota A, et al. Medical treatment of infantile hypertrophic pyloric stenosis: should we always slice the "olive"? J Pediatr Surg 2005;40:1848-51.

5. Yamataka A, Tsukada K, Yokoyama-Laws Y, Murata M, Lane GJ, Osawa M, et al. Pyloromyotomy versus atropine sulfate for infantile hypertrophic pyloric stenosis. J Pediatr Surg 2000;35:338-41; discussion 342.

6. Koike Y, Uchida K, Nakazawa M, Inoue M, Kusunoki M, Tsukamoto Y, et al. Predictive factors of negative outcome in initial atropine therapy for infantile hypertrophic pyloric stenosis. Pediatr Int 2013;55:619-23.

7. Takeuchi M, Yasunaga $\mathrm{H}$, Horiguchi $\mathrm{H}$, Hashimoto H, Matsuda S. Pyloromyotomy versus i.v. atropine therapy for the treatment of infantile pyloric stenosis: Nationwide hospital discharge database analysis. Pediatr Int
2013;55:488-91.

8. Kawahara H, Imura K, Nishikawa M, Yagi M, Kubota A. Intravenous atropine treatment in infantile hypertrophic pyloric stenosis. Arch Dis Child 2002;87:71-4.

9. Singh UK, Kumar R, Suman S. Successful management of infantile hypertrophic pyloric stenosis with atropine sulfate. Indian Pediatr 2001;38:1099-105.

10. Lauriti G, Cascini V, Chiesa PL, Pierro A, Zani A. Atropine Treatment for Hypertrophic Pyloric Stenosis: A Systematic Review and Meta-analysis. Eur J Pediatr Surg https:// doi.org/10.1055/s-0037-1604116.

11. Mercer AE, Phillips R. Question 2: can a conservative approach to the treatment of hypertrophic pyloric stenosis with atropine be considered a real alternative to surgical pyloromyotomy? Arch Dis Child 2013;98:474-7.

12. Meissner PE, Engelmann G, Troeger J, Linderkamp O, Nuetzenadel W. Conservative treatment of infantile hypertrophic pyloric stenosis with intravenous atropine sulfate does not replace pyloromyotomy. Pediatr Surg Int 2006;22:1021-4.

13. WHO WHO | International Classification of Diseases. In: WHO. http://www.who.int/ classifications/icd/en/. Accessed 22 Jun 2018. 14. Yamana H, Moriwaki M, Horiguchi $H$, Kodan M, Fushimi K, Yasunaga H. Validity of diagnoses, procedures, and laboratory data in
Japanese administrative data. J Epidemiol 2017;27:476-82.

15. Feudtner C, Feinstein J, Zhong W, Hall M, Dai D. Pediatric complex chronic conditions classification system version 2: updated for ICD-10 and complex medical technology dependence and transplantation. $B M C$ Pediatr 2014;14:199.

16. Lachin JM. Power and sample size evaluation for the Cochran-Mantel-Haenszel mean score (Wilcoxon rank sum) test and the Cochran-Armitage test for trend. Stat Med 2011;30:3057-66.

17. Armitage P. Tests for linear trends in proportions and frequencies. Biometrics 1955;11:375-86.

18. Kawahara H, Imura K, Yagi M, Kubota A, Okada A. Motor abnormality in the gastroduodenal junction in patients with infantile hypertrophic pyloric stenosis. J Pediatr Surg 2001;36:1641-5.

19. Hall NJ, Eaton S, Seims A, Leys CM, Densmore JC, Calkins CM. Risk of incomplete pyloromyotomy and mucosal perforation in open and laparoscopic pyloromyotomy. J Pediatr Surg 2014;49:1083-6.

20. Kethman WC, Harris AHS, Hawn MT, Wall JK. Trends and surgical outcomes of laparoscopic versus open pyloromyotomy. Surg Endosc 2018;0:1-6. 